\title{
Dor abdominal aguda como manifestação de violência física em lactente: alerta aos pediatras
}

\author{
Acute abdominal pain as a manifestation of physical violence in an infant: alert to pediatricians
}

Patricia Gomes de Souza' ${ }^{1}$ Ana Lúcia Ferreira ${ }^{2}$

\section{RESUMO}

Objetivo: Alertar os pediatras e residentes de Pediatria quanto à possibilidade da ocorrência de violência contra a criança por meio do relato de um caso clínico.

Descrição do caso: Paciente de 18 meses deu entrada à emergência com dor abdominal e vômitos há 48 horas. $\mathrm{O}$ exame abdominal revelou dois orifícios e massa pequena endurecida. $\mathrm{O}$ raio $\mathrm{X}$ de abdome mostrou imagem compatível com três objetos metálicos. Duas agulhas e um prego sem cabeça foram removidos da cavidade abdominal por meio de laparotomia.

Comentários: O diagnóstico foi realizado no segundo atendimento médico, provavelmente por não ter sido aventada a possibilidade de lesão intencional no primeiro. A violência física é um diagnóstico diferencial a ser pensado nos quadros de dor abdominal em crianças. Ressalta-se a importância de aprimorar a formação do residente de Pediatria e dos pediatras em geral para a abordagem da violência contra a criança, de forma que estejam mais preparados para o acionamento da linha de cuidado em situações de violência.

Palavras-chave: síndrome da criança maltratada; dor abdominal; internato e residência; educação médica.

\section{ABSTRACT}

Objective: To alert pediatricians and pediatric residents on the possibility of child abuse by reporting a clinical case.
Case description: An 18 month-old infant was brought to the Emergency Department due to abdominal pain and vomiting for 48 hours. Abdominal examination revealed two holes and a small hardened mass. An abdominal X-ray showed three metallic objects. Two sewing needles and one nail without a head were removed from the abdominal cavity by laparotomy.

Comments: Diagnosis was performed in the second medical care, probably because the intentional injury had not been considered in the first visit. Physical violence is a differential diagnosis to be considered in the presence of abdominal pain in children. It is worth noting the importance of improving pediatric resident training, and also of pediatricians in general, in relation to the approach of child abuse, enabling them to use adequate care in cases of violence.

Key-words: battered child syndrome; abdominal pain; internship and residency; education, medical.

\section{Introdução}

No setor de emergência das unidades de saúde não só é possível suspeitar de casos de violência contra crianças e adolescentes, como é necessário dispensar atenção adequada e acionar serviços de proteção às vítimas ${ }^{(1)}$. Nota-se também que é no pronto-socorro que o médico tem mais oportunidades de contato com diversos casos de violência ${ }^{(2)}$. Entretanto, estudos anteriores mostraram que os médicos têm pouco conhecimento, treinamento e segurança em identificar e tratar a violência na infância ${ }^{(2-4)}$.
Instituição: Universidade Federal do Rio de Janeiro (UFRJ), Rio de Janeiro, RJ, Brasil

${ }^{1}$ Mestre em Medicina pela UFRJ; Médica Pediatra da Secretaria de Estado de Saúde e Defesa Civil do Rio de Janeiro, Rio de Janeiro, RJ, Brasil 2Doutora em Ciências pela Escola Nacional de Saúde Pública Sérgio Arouca da Fundação Oswaldo Cruz; Professora-Associada do Departamento de Pediatria da Faculdade de Medicina da UFRJ, Rio de Janeiro, RJ, Brasil

\author{
Endereço para correspondência: \\ Patricia Gomes de Souza \\ Rua Santa Clara, 308/707 - Copacabana \\ CEP 22041-012 - Rio de Janeiro/RJ \\ E-mail: patigsouza2000@yahoo.com.br \\ Conflito de interesse: nada a declarar \\ Recebido em: 1/2/2012 \\ Aprovado em: 26/4/2012
}


A residência médica em Pediatria é considerada o período de treinamento no qual o médico tem mais oportunidades de adquirir conhecimento e experiência no diagnóstico e no manejo da criança vítima de violência ${ }^{(5)}$, uma vez que os cursos de graduação não contemplam satisfatoriamente este conteúdo ${ }^{(6,7)}$. Apesar disso, muitos residentes concluem seus treinamentos com limitada formação clínica em violência doméstica contra a criança e o adolescente ${ }^{(2-4)}$.

O presente relato teve como objetivo alertar os pediatras e os residentes de Pediatria quanto à possibilidade de ocorrência de violência contra a criança, a partir de um quadro clínico bastante frequente representado pela dor abdominal. Este trabalho foi aprovado pelo Comitê de Ética em Pesquisa do Instituto de Puericultura e Pediatria Martagão Gesteira, da Universidade Federal do Rio de Janeiro (UFRJ).

\section{Descrição do caso}

Paciente de 18 meses, sexo feminino, branca, atendida na emergência pediátrica de um hospital público situado em um município da Baixada Fluminense, no Rio de Janeiro.

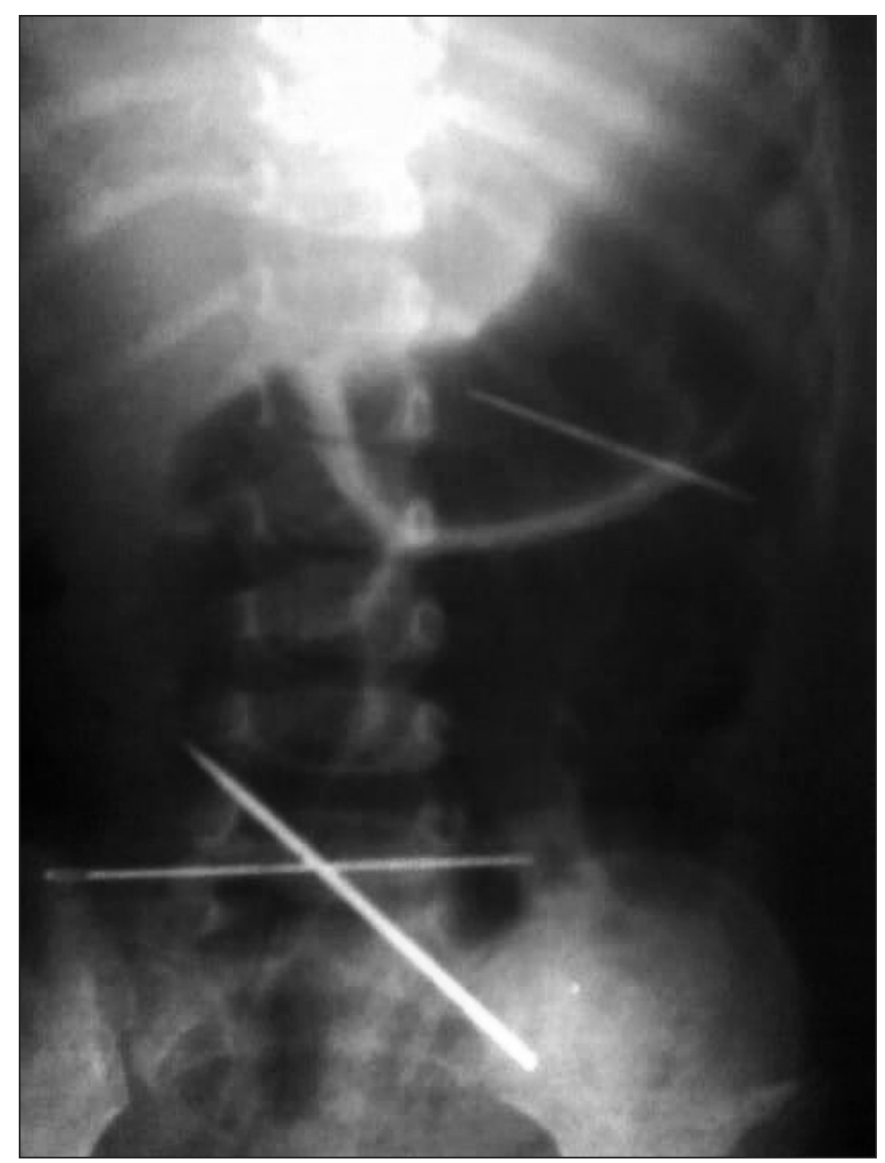

Figura 1 - Raio X de abdome mostrando três objetos metálicos
No mesmo dia, havia sido atendida por um médico pediatra em um pronto-socorro infantil com história de dor abdominal e vômitos há 48 horas, sem febre ou outros sinais e sintomas associados.

No exame físico de entrada, encontrava-se em bom estado geral, afebril $\left(36,7^{\circ} \mathrm{C}\right)$ e hidratada. O exame do abdome evidenciou a presença de dois pequenos orifícios em região de hipogástrio e pequena massa endurecida palpável; os demais sistemas não apresentaram alterações. O hemograma apresentou 20.400 leucócitos, $46 \%$ de segmentados, $50 \%$ de linfócitos, $1 \%$ de bastonetes e $481.000 / \mathrm{mm}^{3}$ plaquetas. $\mathrm{O}$ raio $\mathrm{X}$ de abdome confirmou a presença de três objetos metálicos (Figura 1), e o de tórax estava normal. Após o diagnóstico, foram solicitados os pareceres da Cirurgia Pediátrica, do Serviço Social e do Serviço de Psicologia. A ficha de notificação de violência contra a criança e o adolescente foi preenchida e enviada ao Conselho Tutelar. O caso foi comunicado também à Delegacia de Polícia do bairro, que, após investigação, chegou à conclusão de que o autor da agressão era um vizinho da família, o qual confessou o crime alegando ter inveja pelo fato de a menina ser bonita e amada pelos pais, sendo detido. A paciente foi submetida à laparotomia exploradora, e foram retirados: um prego sem cabeça e duas agulhas (Figura 2). A paciente evoluiu bem, tendo alta hospitalar no sexto dia de internação.

\section{Discussão}

O lactente pertence a uma das faixas etárias mais vulneráveis à violência. No Brasil, as causas externas (acidentes e violência) ocupam a primeira causa de morte na faixa etária de 1 a $19 \operatorname{anos}^{(8)}$. Deve ser dada especial atenção aos casos de crianças com menos de três anos, já que a verbalização é

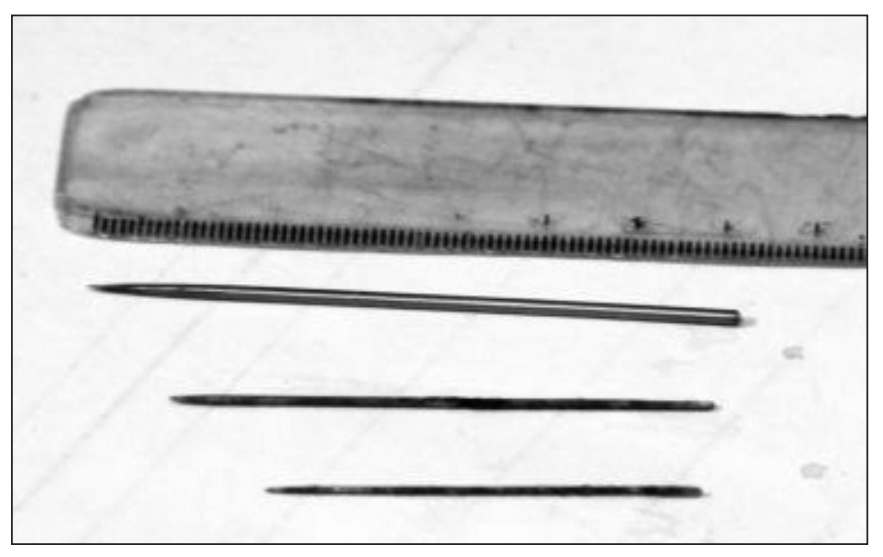

Figura 2 - Duas agulhas e um prego sem cabeça removidos da cavidade abdominal 
inexistente ou ainda precária e as consequências, bem como a exteriorização do sofrimento, dificilmente acontecem de imediato $^{(9)}$. Por esse motivo, o pediatra deve estar atento aos sinais e sintomas que podem representar manifestações de vitimização.

A literatura considera a dor abdominal como uma das manifestações de violência contra crianças e adolescentes, podendo decorrer de ações diretas (no abuso físico ou sexual), mas também expressão de distúrbios emocionais resultantes de maus tratos crônicos ${ }^{(10)}$. As lesões abdominais representam uma importante causa de mortalidade por agravos intencionais na infância ${ }^{(9)}$, considerando a violência como um diagnóstico diferencial fundamental a ser analisado frente a uma criança com queixa de dor abdominal. No caso relatado, houve introdução de corpos estranhos no abdome, prática incomum de violência física contra crianças e, mais raro, o objeto utilizado era uma agulha ${ }^{(10,11)}$. A literatura médica conta com poucos casos descritos desse tipo de lesão e questiona se há influências culturais em tal prática ${ }^{(10-12)}$.

Na mídia brasileira, diferentemente do que acontece na literatura médica, não é raro encontrar o relato da introdução de agulhas em crianças, em geral, lactentes. Em casos recentes, a prática foi associada a rituais religiosos ${ }^{(13-15)}$, o que é um alerta para a influência cultural de violência física no Brasil, embora este não tenha sido o motivo alegado pelo autor da agressão na situação relatada neste artigo. Em tais situações descritas na mídia, o diagnóstico aconteceu após as crianças serem levadas a serviços de saúde com sintomas de dores, vômitos ou sangramentos e, na avaliação radiológica, terem sido encontradas as agulhas.

Neste caso, a criança não foi identificada como vítima de violência em seu primeiro atendimento, sendo feito o encaminhamento a outro serviço por meios próprios, orientação que poderia não ter sido seguida pelo responsável, colocando em risco a vida do paciente.

Os contatos dos profissionais de saúde com crianças e suas famílias são considerados oportunidades para que seja observada a existência de sinais e sintomas resultantes de uma situação de violência. Ao reconhecer e encaminhar adequadamente os casos de violência doméstica contra a criança e o adolescente, o pediatra diminui o risco de revitimização e de suas consequências (prevenção terciária) ${ }^{(9)}$.

As consequências da violência que diretamente atingem a saúde da criança ou do adolescente podem ser imediatas, de médio e de longo prazo ${ }^{(16)}$. As imediatas são mais facilmente identificadas, pois tendem a deixar marcas visíveis na pele ou no sistema osteoarticular; no entanto, aquelas de médio e longo prazo podem se manifestar como distúrbios psicossomáticos, gastrintestinais crônicos e remitentes, ansiedade ou depressão, agressividade, timidez, comportamento abusivo (poderão se tornar autores de agressão futuramente), além de distúrbios do sono e do apetite ${ }^{(17)}$.

Apesar de a violência doméstica contra a criança e o adolescente não ser um problema novo enfrentado pelos profissionais de saúde no seu dia-a-dia de trabalho, ainda existem dificuldades em sua identificação, fundamental para a prevenção e o manejo adequado da violência ${ }^{(18)}$.

Os motivos para as dificuldades de identificação são vários, e se interligam. O primeiro diz respeito ao nível insuficiente de informação de que os profissionais de saúde dispõem sobre o tema ${ }^{(19)}$, uma vez que o estudante sai da faculdade sem compreender o fenômeno da violência; por outro lado, a residência médica, igualmente, não oferece espaço para esse conhecimento $^{(7)}$. O segundo refere-se ao desconhecimento da lei por parte desses profissionais ${ }^{(19)}$, os quais, muitas vezes, não se sentem corresponsáveis pela proteção das crianças, contrariando o que é recomendado pelo Estatuto da Criança e do Adolescente. Cabe ressaltar que outro aspecto que dificulta a identificação dos casos de violência é o processo de atendimento, geralmente condicionado pelas limitações estruturais do serviço, como uma prática unicamente socorrista, o que pode ter acontecido no caso relatado. Dificilmente as verdadeiras causas dos agravos são investigadas, o que contribui para seu ocultamento e repetição ${ }^{(18)}$.

Nota-se que, mesmo entre pediatras experientes, o relato da dificuldade na identificação de maus tratos está presente. Bannwart e Brino ${ }^{(20)}$, ao estrevistarem pediatras com tempo médio de experiência profissional de 22 anos, apontaram que 75\% dos participantes relataram dúvida quanto à existência da suspeita de violência.

As maiores dificuldades relatadas por profissionais dizem respeito à identificação e ao encaminhamento em situações de violência doméstica contra a criança, além da limitação para sua resolução em função de medos implícitos na relação profissional-família-comunidade ${ }^{(2,5)}$. O medo talvez seja um possível bloqueador do aprendizado e, consequentemente, do desempenho que deve ser trabalhado para que se experimentem novos resultados ${ }^{(2)}$. Para tanto, a formação do residente deve prepará-lo para essa abordagem.

Em um estudo realizado com residentes de Pediatria, o medo foi evidenciado como impedimento, limitando a possibilidade de reconhecer a criança ou o adolescente em sua alteridade. Os entrevistados expressaram medo e sentimentos como pré-julgamentos, desinteresse e negação. Mais da 
metade citou dificuldades técnicas (como fazer a anamnese, diagnosticar, romper o pacto do silêncio e as omissões), considerando a própria formação médica insuficiente ${ }^{(2)}$. Tais fatos sugerem que o treinamento na residência não tem focado novos desenvolvimentos no campo da violência infantil ${ }^{(3)}$.

A dificuldade do ensino das questões da violência doméstica contra a criança e o adolescente é comum e o fato de haver protocolos e novas propostas não tem garantido um maior compromisso do pediatra ${ }^{(2)}$. Poucos abordam a questão da violência durante a graduação, o que traz a necessidade urgente de incluir o tema nos currículos e de capacitar os profissionais graduados ${ }^{(21)}$.

Evidentemente, como mostram alguns estudos, a base do conhecimento dos residentes tem sido considerada significativamente fraca e com necessidade de melhora $^{(3,22)}$, o que poderia ser levado a cabo por meio do aumento nas oportunidades de treinamento ${ }^{(3)}$. Mesmo os programas sem experiências clínicas na violência infantil podem preparar melhor seus residentes, enfatizando e promovendo a qualidade didática de ensino ${ }^{(3)}$ com rodízios em estágios de serviços que atendam crianças e adolescentes vítimas de violência, além de um minicurso intensivo ${ }^{(4)}$.

Ressalta-se a importância dos preceptores de levar em consideração a experiência fornecida aos seus residentes e sugerir que um currículo padronizado e estruturado possa ser desenvolvido para atender às necessidades de ensino ${ }^{(4)}$. A implementação de um currículo padronizado em violência contra a criança poderia incluir a reformulação do conteúdo do treinamento médico baseado na avaliação do reconhecimento, prevenção e do manejo da violência infantil, usar um formato capaz de criar novos modelos de programas com currículos baseados em violência infantil e publicar os resultados alcançados de forma a reforçar êxitos e gerar novas mudanças ${ }^{(23)}$. Deve-se acrescentar ao currículo referência bibliográfica e fotos educativas sobre o tema, discussão de casos

\section{Referências bibliográficas}

1. Moraes SR, Ferreira AL. Como reconhecer e atender às crianças vítimas de violência na emergência. Rev Científica FMC [homepage on the Internet]. 2008;3:2-7. [cited 2012 Mar 30]. Available from: http://www.fmc.br/revista/ revistaCientificaVolume3Numero1.pdf

2. Bourroul MLM, Rea MF, Botazzo C. Pediatric residents confronted with domestic violence against children and adolescents. Interface - Comunic Saude Educ 2008;12:737-48.

3. Narayan AP, Socolar RR, St Claire K. Pediatric residency training in child abuse and neglect in the United States. Pediatrics 2006;117:2215-21. clínicos, protocolos de atendimento para violência doméstica contra a criança e o adolescente, além de outros instrumentos necessários para a educação do residente ${ }^{(24)}$.

Nos serviços de saúde, algumas propostas podem facilitar o trabalho dos profissionais no enfrentamento da violência doméstica contra a criança e o adolescente: cursos de capacitação para atuação do profissional, palestras educativas para as mães, pais e/ou responsáveis pela criança nos setores de Puericultura e Pediatria e divulgação de protocolos de atendimento para as vítimas de violência na rede de saúde ${ }^{(19)}$.

A fim de proteger as crianças e os adolescentes vítimas de violência, é importante garantir que os profissionais de saúde sejam capazes de desenvolver as habilidades e o conhecimento para seguir, de forma eficaz, o passo a passo da linha de cuidado para a atenção à saúde de crianças em situação de violência (acolhimento seguido pelo atendimento, notificação e seguimento na rede de cuidado e de proteção social $)^{(9)}$. Essa linha de cuidado não foi acionada no primeiro atendimento da criança no caso relatado, colocando-a em risco de novas agressões, especialmente por tratar-se de um lactente.

Muitos serviços de emergência são campos de estágio supervisionado para médicos residentes. Sendo assim, há um caminho de mão dupla no qual todos se beneficiam com uma formação sólida no atendimento a crianças vítimas de violência: um pediatra com habilidade para prestação de tal atendimento poderá transmitir seus conhecimentos aos médicos residentes, os quais, por sua vez, também podem ser a fonte de informação atualizada para os preceptores que porventura não tenham tido a oportunidade de adquirir este conhecimento. Ao incluir a violência de forma consistente e segura no rol de possibilidades do diagnóstico diferencial para os mais variados quadros clínicos, profissionais e família estarão mais aptos a traçarem estratégias conjuntas de proteção às crianças.
4. Ward MG, Bennett S, Plint AC, King WJ, Jabbour M, Gaboury I. Child protection: a neglected area of pediatric residency training. Child Abuse Negl 2004;28:1113-22

5. Accreditation council for graduate medical education [homepage on the Internet]. Program Requirements for Graduate Medical Education in Pediatrics [cited 2012 March 30]. Available from: http://www.acgme.org/acWebsite/ downloads/RRC_progReq/320_pediatrics_07012007.pdf

6. Almeida EC. Violência doméstica: um desafio para a formação do pediatra [tese de mestrado]. Rio de Janeiro (RJ): Universidade Estadual do Rio de Janeiro; 1998. 
7. Sociedade Brasileira de Pediatria. Violência é covardia. E pode ser preventiva no consultório. SBP notícias 2011;62:24-5.

8. Brasil. Ministério da Saúde. Secretaria de Vigilância em Saúde. Departamento de Análise de Situação de Saúde. VIVA - Vigilância de Violência e Acidentes 2006-2007. [Série G. Estatística e Informação em Saúde]. Brasília: Ministério da Saúde; 2009.

9. Brasil. Ministério da Saúde. Secretaria de Atenção à Saúde. Departamento de Ações Programáticas Estratégicas. Linha de cuidado para a atenção integral à saúde de crianças, adolescentes e suas famílias em situação de violências: orientação para gestores e profissionais de saúde. Brasília: Ministério da Saúde; 2010.

10. Gadodia A, Seithbhalla A. Re: migratory foreign body of neck in a battered baby: a case report. Int J Pediatr Otorhinolaryngol 2010;74:432-3.

11. $\mathrm{Ng} \mathrm{CS}$, Hall CM, Shaw DG. The range of visceral manifestations of nonaccidental injury. Arch Dis Child 1997;77:167-74.

12. Amirjamshidi A, Ghasvini AR, Alimohammadi M, Abbassioun K. Attempting homicide by inserting sewing needle into the brain Report of 6 cases and review of literature. Surg Neurol 2009;72:635-41.

13. Meio Norte [homepage on the Internet]. Garota foragida era vitima de rituais de magia negra [cited $2011 \mathrm{Dec} 30]$. Available from: http://www.meionorte.com/ noticias/policia/garota-foragida-era-vitima-de-rituais-de-magia-negra-118281.html

14. Baptista R. Padrasto confessa ter enfiado quase 50 agulhas em menino na Bahia, diz polícia. Agência Folha 2011.

15. Sant'Anna P. Outro caso com agulhas. Blog do Paulo Sant'Anna 2011.
16. Reichenheim ME, Hasselmann MH, Moraes CL. Consequences of family violence to the health of children and adolescents: contributions to action proposals. Cienc Saude Coletiva 1999;4:109-21.

17. Kashani JH, Daniel AE, Dandoy AC, Holcomb WR. Family violence: impact on children. AACAP 1992;31:181-9.

18. Deslandes SF. Care of children and adolescents suffering domestic violence: analysis of a service. Cad Saude Publica 1994;10 (Suppl 1):177-87.

19. Brasil. Ministério da Saúde. Secretaria de Atenção à Saúde. Departamento de Ações Programáticas Estratégicas. Violência doméstica contra crianças e adolescentes. Brasília: Ministério da Saúde; 2002.

20. Bannwart TH, Brino RF. Difficulties to identify and report cases of abuse against children and adolescents from the viewpoint of pediatricians. Rev Paul Pediatr 2011;29:138-45.

21. Gonçalves HS, Ferreira AL. Health prefessionals' reporting of family violence against children and adolescents. Cad Saude Publica 2002;18:315-9.

22. Ramos ML, Silva AL. Study about domestic violence against children in primary health care units in São Paulo - Brazil. Saude Soc [homepage on the Internet]. 2011;20 [cited 2012 Mar 30]. Available from: http://www.scielo.br/scielo. php?script=sci_arttext\&pid=S0104-12902011000100016\&lng=en\&nrm=iso

23. Botash AS. From curriculum to practice: implementation of the child abuse curriculum. Child Maltreat 2003;8:239-41.

24. Starling SP, Boos S. Core content for residency training in child abuse and neglect. Child Maltreat 2003;8:242-7. 